\title{
Philosophiques
}

\section{Husserl, Brentano et la psychologie descriptive}

\section{Fabien Cayla}

Volume 20, numéro 2, automne 1993

Perspectives sur la phénoménologie et l'intentionnalité

URI : https://id.erudit.org/iderudit/027230ar

DOI : https://doi.org/10.7202/027230ar

Aller au sommaire du numéro

Éditeur(s)

Société de philosophie du Québec

ISSN

0316-2923 (imprimé)

1492-1391 (numérique)

Découvrir la revue

Citer cet article

Cayla, F. (1993). Husserl, Brentano et la psychologie descriptive. Philosophiques, 20(2), 347-361. https://doi.org/10.7202/027230ar d'utilisation que vous pouvez consulter en ligne.

https://apropos.erudit.org/fr/usagers/politique-dutilisation/ 


\title{
HUSSERL, BRENIANO EI LA PSYCHOLOGGE DESCRIPTIIVE
}

\author{
par \\ Fabien Cayla
}

Dans sa propre contribution au volume consacré à Husserl dont il est l'éditeur, "Husserl's Perceptual Noema »" Hubert Dreyfus a remarquablement mis en évidence les difficultés que pose la notion de sens remplissant :

I...I alors que les actes signifiants peuvent être conçus comme opaques Ipour adopter la terminologie de Russelll et donc toujours corrélés à un sens, les actes remplissants - en vertu de leur fonction de représentation de l'état de choses lui-même - ne peuvent jamais être interprétés comme référentiellement opaques [...] Cette différence de fonction entre les actes signifiants et remplissants est à ce point fondamentale qu'il demeure douteux qu'il y ait en quelque façon un sens remplissant ${ }^{2}$.

Dreyfus remarque en note ${ }^{3}$ que l'analyse des termes de perception que propose Chisholm dans Perceiving ${ }^{4}$ semble contester que les actes remplissants soient toujours interprétés comme transparents. Mais c'est que le sens propositionnel des verbes de perception implique toujours pour Chisholm des locutions épistémiques, qui sont opaques. Dans un texte récent, Chisholm précise que sa définition d'un sens non propositionnel des verbes de perception ( $\ll S$ perçoit $x$ » par opposition à «S perçoit que $x$ est $F \gg)$ n'utilisait aucune locution psychologique et intentionnelle :

[...] mon intention [...] était d'éviter l'emploi de tout concept « représentationnel » [intentionnell et de ne faire référence qu'à une relation causale entre les états du sujet percevant et l'objet ou les objets de perception. Mais j'ajoutais qu'ordinairement nous ne dirions pas qu'une personne $S$ perçoit une chose $\mathrm{x}$ à moins qu'elle

I. Hubert Dreyfus (dir.), Husserl, Intentionality and Cognitive Science, Cambridge Mass., M.I.T. PTess, 1982.

2. Ibid., p. I03.

3. Ibid., p. 325

4. R. Chisholm, Perceiving, Ithaca, Cornell University Press, 1957. 
ne considère ltakesl x comme ayant une certaine propriété - et cette considétation est, bien sûr, intentionnelle ${ }^{5}$.

Dans la note en question, Dreyfus indique que

[...] si l'on pouvait interpréter percevoir comme « considérer » k taking» ou « voir »] que quelque chose est le cas, plutôt que comme voir simplement quelque chose, la thèse intentionnaliste pourrait être sauvée. Ceci semble être précisément la tactique de Husserl lorsqu'il généralise sa théorie de la signification à la perception ${ }^{6}$.

La thèse intentionnaliste, développée par Brentano et son école ${ }^{7}$, consiste à soutenir que les actes mentaux ou psychologiques ont une relation à un contenu |Beziehung auf einen Inhalt] ou une direction vers un objet [Richtung auf ein Objekt] ${ }^{8}$. Bien que Brentano tienne apparemment ces formulations pour equivalentes, il convient pourtant de les distinguer, si l'on veut réconcilier celui-ci avec son école - en particulier avec Marty et Meinong -, mais aussi avec lui-même - c'est-à-dire le Brentano d'avant et d'apres 1905. En acceptant de distinguer relation intentionnelle à un contenu et direction intentionnelle vers un objet, on peut alors éviter l'emploi - qui prête à confusion - des expressions « objet immanent » et « objet transcendant ». On peut formuler la thèse intentionnaliste de la manière suivante:

TI : Les actes psychologiques ont une direction vers un objet en vertu du fait qu'ils ont une relation à un contenu.

Dans la mesure où un acte n'est pas, à proprement parler, reliéà un contenu, (TI) peut être reformulée ainsi :

$\mathrm{TI}^{*}$ : Les actes psychologiques ont une direction vers un objet en vertu du fait qu'ils ont un contenu.

Mais $\left(\mathrm{TI}^{*}\right)$ omet, avec la relation intentionnelle, l'agent ou la personne. En admettant, avec Brentano et Chisholm, la primauté du personnel, nous proposons de formuler ainsi la thèse intentionnaliste :

5. Roderick Chisholm, "Replies », dans R. Bogdan, Roderick Chisholm, Dordrecht, Kluwer Academic, ig86, p. 207.

6. H. Dreyfus, op. cit., p. 326.

7. En particulier par Marty, Meinong, Mailly, Husserl et Twardowski.

8. Brentano, Psychologie vom empirischen Standpunkt, vol. I. Hambourg. Felix Meiner, 1973. P. I24, tr. fr. M. de Gandillac, Paris, Aubier-Montaigne, 1944, p. 102. 
$\mathrm{TI}^{* *}$ : Les agents ou personnes peuvent diriger leurs pensées vers un objet en vertu du fait que ces pensées ont un contenu.

Ce qui est essentiel pour l'intentionnalité, c'est donc le sujet pensant et le contenu. La question que je souhaite poser est la suivante : dans quelle mesure les sens remplissants husserliens et plus généralement, l'analyse de la perception - peuvent-ils rentrer dans un tel cadre ? La conclusion du présent article incline à une réponse négative, mais qui ne sera pas directement argumentée. Dans la première section, je tenterai de clarifier certains points de la doctrine brentanienne, dans la deuxième section, j'essaierai de déterminer l'apport novateur de Husserl, et j'indiquerai dans la dernière section certains parallèles contemporains, en suivant, quoique dans une intention opposée, les rapprochements proposés par Dreyfus dans son introduction éditoriale à Husserl, Intentionality and Cognitive Science.

Lorsque Brentano, dans la Psychologie de 1874 , définit l'intentionnalité par la direction vers un objet ou la relation à un contenu, il ajoute qu'il ne faut pas entendre par là une chose [Realität $]^{9}$. Mais par « Realität », Brentano n'entend pas une chose existante, et divers auteurs - A. Marras, L. McAlister, Chisholm et Dummett - ont proposé de rendre « Realität » par «chose » plutôt que par « réalité ». En effet, selon la doctrine médiévale de l'esse intentionale - que reprend Brentano -, un acte mental possède ou contient un objet, qui est donc immanent à l'acte :

Ce qui caractérise tout phenomène psychique, c'est ce que les scolastiques du Moyen-Âge nommaient l'inexistence intentionnelle (ou encore mentale) d'un objet (die intentionale (auch wohl mentale) Inexistenz eines Gegenstand ${ }^{\text {IO }}$.

L'inesse mental d'un objet - ou son esse objectif - interdit de lui confèrer une existence transcendante ${ }^{\mathrm{II}}$. Même si, par conséquent, le contenu ou l'objet d'un acte mental était une chose, il ne s'agirait pas d'une chose existant hors de l'esprit. Dummett, qui a

9. Ibid., p. I24, tr. fr., p. IO2.

10. Ibid., p. 124, tr. fr., p. 102.

II. Voir sur ce point Barry Smith, « The Soul and its Parts », dans Brentano Studien, I, 1988, P. 75-87; aussi « Brentano and Marty », dans K. Mulligan (dir.), Mind, Meaning and Metaphysics, Dordrecht, Kluwer Academic, 1990, p. Irz sq. 
pourtant parfaitement exposé ce point ${ }^{\mathrm{I2}}$, écrit ailleurs hâtivement que :

[...] dans sa thèse positive la plus célèbre, il [Brentano] soutient que les actes de conscience sont caractérisés par leur intentionnalité, ce qui signifie qu'ils sont orientés vers des objets extérieurs ${ }^{\mathrm{I3}}$.

Si Brentano a fini par soutenir quelque chose de ce genre à la fin de sa vie - ce que les textes publiés ne permettent pas d'affirmer $^{\mathrm{I}}$ - ce n'est en tout cas pas ce qu'il soutient dans la Psychologie de 1874 ni dans la Deskriptive Psychologie de I889, dans laquelle il parle de la relation intentionnelle à un objet immanent [intentionale Beziehung auf ein immanentes Objektl ${ }^{15}$.

Avant 1905, donc, Brentano soutenait que le contenu ou l'objet d'un acte psychologique pouvait n'être pas une chose. L'opposition est ici entre les choses singulières et les abstracta, comme les propositions et les états de choses, entre les realia et les irrealia. «Ein Realität» signifie donc un individu au sens de Nelson Goodman $^{\mathrm{I} 6}$ : il y a (si l'on peut employer cette tournure meinongienne) des individus non existants qui sont objets d'attitudes intentionnelles. Mais en 1874 , Brentano admettait que les irrealia peuvent aussi être objets d'attitudes intentionnelles. A partir de I905, Brentano n'admet plus qu'une ontologie d'individus ${ }^{17}$. Il

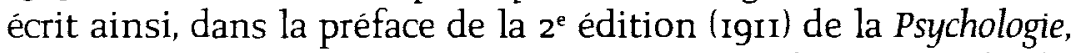
qu'il n'est plus d'avis « qu'une relation mentale leine psychische

12. M. Dummett, «Thought and Perception », dans Bell \& Cooper (dir.), The Analytic Tradition, Oxford, Blackwell, 1990, p. $84-85$.

13. M. Dummett, Ursprünge der analytischen Philosophie, Frankfort, Suhrkamp, 1988, p. 39. Voir également Føllesdal, «Brentano and Husserl on Intentional Objects », dans H. Dreyfus, op. cit.. p. 32, auquel Dummett fait référence. Selon Føllesdal, Brentano a fini par renoncer à la thèse de l'immanence des objets intentionnels.

14. Voir la lettre a Marty du $17 / 03 / 1905$, citée plus loin, dans laquelle Brentano maintient que l'objet de pensée est immanent.

15. H. Dreyfus, op. cit., p. I31.

16. Voir par exemple, N. Goodman, Of Mind and other Matters, Cambridge Mass., Harvard University PTess, r984, p. 50-I.

17. M. Dummett, « Thought and Perception », op. cit., p. 86, remarque excellemment que « Brentano employait le terme "chose" selon l'usage alors généralement courant dans le langage philosophique allemand, c'est-à-dire pour signifier un particulier concret qui pouvait être, pourlui, soit matériel, soit spirituel : une substance au sens de Descartes, donc. Il avait soutenu à l'origine que l'objet d'un acte mental n'avait pas lieu dêtre une chose en ce sens ; ll avait en particulier admis quill pouvait être ce quil désignait comme "contenu", c'est-à-dire le contenu d'une proposition. Selon sa conception ultérieure, l'objet d'un acte mental ne pouvait être que quelque chose de "real", c'est-à-dire, non pas nécessairement réel au sens d'existant actuellement, mais du type d'une chose "thing-like": tous les actes mentaux doivent avoir pour objets des particuliers concrets. » 
Beziehungl puisse jamais avoir pour objet quoi que ce soit d'autre qu'une chose singulière [reales] $\gg^{18}$. Brentano doit donc analyser les pensées en apparence à propos d'entia irrealia de telle façon qu'elles n'aient plus pour objets que des individus (physiques ou spirituels) - ou plutôt des choses qui, si elles existaient, seraient des individus.

Il en résulte une modification de la théorie du jugement. Croyances et jugements ne peuvent plus porter sur des entia irrealia comme les propositions et états de choses. D'où la théorie non propositionnelle du jugement, qui n'implique que la relation d'un sujet pensant à un individu ou, dans le cas d'une prédication, une relation à deux individus dont l'un est partie de l'autre. Substances et accidents étant des entia realia, l'analyse proposée supprime « le besoin apparent de supposer que les choses individuelles sont en relation avec des objets abstraits $\gg^{19}$.

Dans les textes complémentaires de la Psychologie, qui datent de igiı, Brentano pense résoudre le problème de la relation intentionnelle par la distinction du modus rectus et du modus obliquus ${ }^{20}$. Si je pense à une personne qui pense à un centaure, je pense à la personne en modo recto et au centaure en modo obliquo. Mais cette stratégie ne suffit pas, une relation doit unir deux choses singulières existantes alors que les relations intentionnelles ont pour relatum des choses singulières qui peuvent ne pas exister. Brentano, dès les premières lignes des Remarques complémentaires, notant que les références mentales sont telles que l'un des relata peut manquer à exister, indique qu'elles doivent être dites, non pas des relations, mais plutôt des quasi-relations:

Si quelqu'un pense à quelque chose, la personne qui pense doit certainement exister, mais l'objet de sa pensée n'a nul besoin

18. Le lecteur français serait aisément induit en erreur puisque la traduction française rend « Reales » par « réalité effective », sans doute par inadvertance, puisque la préface du traducteur précise en note que Reale s'oppose à Wirkliches : « Aussi traduisonsnous ce demier terme par effectif chaque fois quil sera nécessaire de marquer l'opposition (Pégase, par exemple, est objet réel de conscience, mais il n'existe pas effectivement » (tr. M. de Gandillac, p. 13). Voir Chisholm, Brentano and Intrinsic Value, Cambridge Mass., Cambridge University Press, 1986. p. 9 : "Il ne serait pas correct de traduire ens reale par "chose réelle" ou "chose actuelle". Ies pensées sur les licomes ou les sirenes, aurait dit Brentano, sont des pensées sur des entia realia. Mais ce ne sont pas des pensées sur des choses actuelles. La meilleure traduction pour ens reale est "non individu". »

19. R. Chisholm, ibid., p. I6.

20. Il s'agit des « Remarques complémentaires 》, Von der Klassifikation der psychischen phänomene. 
d'exister [...] On pourrait alors se demander si nous avons vraiment affaire ici à quelque chose de seulement similaire à une relation, et que nous pourrions nommer de manière plus appropriée quasirelationnel |etwas Relativlisches| ${ }^{2 \mathbf{I}}$.

Cette analyse remet-elle en cause la thèse de l'immanence des objets intentionnels ? Il ne semble pas. L'ontologie des realia n'implique pas que les reales soient des choses existantes, et encore moins des choses physiques ${ }^{22}$; ce sont seulement des individus, des particuliers concrets donnés ou actuellement pensés : seuls les non-individus ne peuvent être objets des attitudes intentionnelles.

C'est ce que me paraît indiquer ce passage d'une lettre à Marty :

Lorsque je parlais d'« objet immanent », j'ajoutais l'expression « immanent » afin d'éviter les méprises, du fait que beaucoup entendaient par « objet » ce qui est en dehors de l'esprit. Par contraste, je parlais d'un objet de la représentation, qui est également ce à quoi elle renvoie lorsqu'il n'y a rien d'extérieur à l'esprit qui y correspond. Je n'ai jamais été de l'opinion que l'objet immanent $=$ « objet représenté » lvorgestelltes Objektl. La représentation n'a pas « la chose représentée », mais plutôt « la chose », ainsi, par exemple, la représentation d'un cheval lal non «cheval représenté », mais plutôt « cheval » comme objet limmanent, c'est-à-dire le seul objet à proprement parlerl ${ }^{23}$.

Aquila $^{24}$, en commentant des passages semblables cités par Krauss dans l'édition de Wahrheit und Evidenz, tente d'évacuer la notion d'immanence parce qu'il la suppose impliquer l'existence des objets de pensée, et donc des ficta. Il conjecture donc que Brentano veut distinguer entre, disons, le centaure qui peut être objet de pensée - le centaure comme objet immanent-et les pensées de centaures, c'est-à-dire les centaures représentés, ou représentations de centaures, lesquelles existent en tant que pensées. Il s'ensuivrait, selon Aquila, que :

I... à moins qu'il puisse établir une distinction entre un centaure à quoi l'on peut penser lobjet immanentl et une «pensée-de-

21. Brentano, op. cit., vol. II, p. 134, tr. fr. p. 268.

22. D. Føllesdal, op.cit., P. 32 parle de « full-fledged physical object ».

23. Brentano, « Lettre du $17 / 03 / 1905$ », dans Die Abkehrvom Nichtrealen. Hambourg. Felix Meiner Verlag, 1966, p. II9-120.

24. R. Aquila, Intentionality, University Park, Pennsylvania State University Press, 1977. p. $16-17$. 
centaure » lvorgestelltes Objektl, Brentano est de fait engagé à assumer l'existence de tous les centaures auxquels on peut penser ${ }^{25}$.

Mais la distinction entre objet immanent et vorgestelltes Objekt ne porte pas sur un supposé engagement ontologique envers des objets fictifs. Elle concerne en fait le contenu des pensées. Si ce contenu était celui d'un objet représenté, il s'agirait là d'un ens irreale, un objet abstrait exclu par la dernière ontologie de Brentano.

Le contenu d'une pensée ne peut être qu'un ens reale, c'est-àdire un individu, eine Realität, qui n'a pas besoin d'être existant.

Même si la quasi-relation intentionnelle ne peut, en fin de compte, selon le dernier Brentano, valoir qu'entre des choses singulières, il ne peut s'agir d'un rapport de l'agent au monde, tel que lillustre par excellence la perception. Comme l'a parfaitement vu Putnam, c'est Husserl qui interprétera en ce sens l'intentionnalité, interprétation qui allait connaître une fortune considérable :

Brentano lui-même n'a jamais, à ma connaissance, employé le terme « intentionnalité » ni le terme d'« inexistence intentionnelle » pour désigner la relation entre l'esprit et le monde réel, ainsi que les philosophes en sont venus à employer le terme « intentionnalité » après Husserl. C'est Husserl, non Brentano, qui a vu dans l'intentionnalité du mental un moyen de comprendre comment l'esprit et le monde sont reliés et comment il se fait que dans les actes de conscience nous en arrivions à être dirigés vers un objet $^{26}$.

C'est sans doute parce que Brentano n'a jamais conçu l'intentionnalité de cette façon qu'il a constamment méconnu la distinction proposee par Meinong et Twardowski entre contenu et objet d'un acte mental ${ }^{27}$. Au contraire, Husserl a toujours admis cette distinction. Il nommera d'abord matière le contenu d'un acte, puis, avec l'introduction du noema, sens [Sinn] : le contenu d'un acte est un sens :

25. Ibid.

26. H. Putnam, Representation and Reality, Cambridge Mass., M.I.T. Press, 1988, note $\mathrm{I}$ du chap. I, tr. fr. Claudine Engel-Tiercelin, Paris, Gallimard, 1990, p. 21 .

27. Concernant l'attribution à Twardowski de cette distinction, voir D. Jacquette, "The Origins of Gegenstandstheorie », Brentano Studien, 3, 1990/199I. p. 178 : «Twardowski, in Zur Lehre vom Inhalt und Gegenstand der Vorstellungen, que lhistoire reçue de la philosophie intentionnaliste aime à créditer comme étant la source de la distinction de Meinong entre acte, contenu et objet de représentation, se référe à Höffler et Meinong comme source d'inspiration pour son traité de $1874 \%$. Jacquette remarque à juste titre que 
(...) dont nous disons que, en lui, la conscience se rapporte à un objet comme « sien » Igegenstandliches als das « seine » Beziehtl |...I Tout noème

a un « contenu », à savoir son « sens», et se rapporte par lui à «son » objet ${ }^{28}$.

Comme l'indique Barry Smith, Brentano a toujours été « parfaitement clair concernant l'opposition entre l'acte et l'objet (immanent) - sa doctrine de l'intentionnalité n'est, en fin de compte, rien d'autre qu'une tentative de préciser la relation entre les deux $\gg^{29}$.

\section{\|}

Si Husserl respecte la lettre de la distinction entre contenu et objet d'un acte intentionnel, il l'annule cependant dans les faits avec l'introduction des sens remplissants, qui présentent la chose même dans son incarnation propre, la perception devenant ainsi le lieu privilégié de l'intentionnalité. Le modèle inaugural des Logische Untersuchungen est en effet celui de la Bedeutungsintention, laquelle vise à son remplissement dans un acte intuitif de ce qui, dans la seule intention, est visé à vide [Leermeinungl. Parce que la conscience est donatrice d'objets sur le même mode intuitif, les différences catégorielles des objets visés tendent à passer au second plan, de sorte que la structure des actes d'appréhension de la signification est généralisée et appliquée aux actes de perception. Actes d'intention et actes de remplissement peuvent ainsi se superposer dans tous les domaines de la conscience d'objets.

La sixième Recherche caractérise l'intention comme:

[...] classe de vécus intentionnels qui [possèdent] la propriété spécifique de pouvoir fonder des rapports de remplissement [Erfuillungsverhältnissel. Dans cette classe entrent tous les actes appartenant à la sphère [...I du logique, parmi lesquels également les actes qui,

la traduction anglaise de l'ouvrage de Twardowski qu'a proposée Reinhardt Grossmann rend incorrectement «beziehen » par « viser » $\mathrm{k}$ intends 》) lorsque l'auteur écrit "Cest l'une des propositions les plus conmues de la psychologie, difficilement contestée par quiconque, que tout phénomene mental se rapporte lbeziehtl a un objet immanent », Jacquette, ibid., p. 200. п. 14. Le passage en question est au dèbut de l'ouvrage, Twardowski, Zur Lehre vom Inhalt und Gegenstand der Vorstellungen, Vienne, Philosophia Verlag, 1982, p. 3.

28. Husserl, Ideen zu einer reinen Phänomenologie, La Haye, Martinus Nijhoff, I950, § 129. P. 3I6, tr. fr., Paris, Gallimard 1952, p. 436.

29. B. Smith, The Soul and its Parts, op. cit., p. 82 . 
dans la connaissance, sont destinés au remplissement d'autres intentions lzur Erfülung anderer Intentionenl, les intuitions ${ }^{30}$.

L'indétermination de l'intention appelle un remplissement actuel, selon un certain champ de remplissement possible - relations de remplissement qui seront ultérieurement nommées «validations ». Il faut en effet distinguer entre un concept étroit et un concept large d'intention :

L'expression d'intention représente le caractère propre des actes sous l'image de la visée d'un but [...] cette image ne convient pas également bien à tous les actes [...] les remplissements sont eux aussi bien des actes donc ils sont aussi des «intentions», quoique [du moins en générall ils ne soient pas cette fois des intentions dans ce sens plus étroit qui renvoie à un remplissement correspondant ${ }^{3 \mathrm{I}}$.

Le contenu d'une intention de signification ne peut pas en effet être identique à celui d'une intention de remplissement : la première est visée [Meinungl de l'objet, et cette référence, non réalisée dans la simple intention de signification, s'actualise lorsque celleci se remplit.

On doit alors distinguer entre un acte conférant le sens Isinngebendem Aktl et un acte remplissant le sens [sinnerfullendem $A k t]$. Actes intentionnels - au sens étroit - et actes remplissants fusionnent donc dans la présence intuitive de l'objet, de sorte qu'au sens intentionnel est associé le sens remplissant. L'objet est alors donné dans l'acte remplissant tel qu'il est visé dans l'acte intentionnel. Toutefois, l'objet comme tel n'est pas l'objet donné - ni, a fortiori, l'objet visé : nous avons donc i) le sens intentionnel, iI) le sens remplissant, et III) l'objet lui-même, réel ou fictif. La connaissance est alors définie comme coïncidence dans l'évidence des actes significatifs et des actes intuitifs, toute pensée pouvant devenir intuitive - la pensée intuitive étant la perfection de la pensée $^{32}$. La prévalence accordée aux rapports de remplissement permet de comprendre pourquoi la perception allait devenir chez Husserl le lieu privilégié de l'intentionnalité. On doit faire le partage entre la perception adéquate (ou intuition au sens étroit) et la perception inadéquate. Dans la première:

30. E. Husserl, Logische Untersuchungen : Zweiter Band, La Haye, Martinus Nijhoff, I984, $\S$ Io, p. 572.

31. Ibid., V, § 13. p. 39I, tr. fr. p. 18I.

32. Ibid., II, § 23-24, p. 168-170, tr. fr. p. 191-197. 
[... l'intention de perception [wahmehmende Intention] est orientée exclusivement sur un contenu qui lui est réellement présent lauf einen ihr wirklich präsenten Inhalt gerichtet istl l...]

alors que la seconde est

[...] simplement présomptive [...] l'intention ne trouve qu'un remplissement partiel [...] dans le contenu présentélim präsenten Inhalt] et, par celui-ci, renvoie au-delà du donné33.

C'est dans cette présence intuitive de la chose que la distinction du contenu et de l'objet paraît annulée. Contrairement à ce qui se produit dans la perception adéquate, dans la perception inadéquate,

[...] l'objet visé ou, si l'on veut, l'objet intentionnel lder intendierte oder wenn man will, der intentionale Gegenstandl n'est pas immanent à l'acte dans son occurrence lerscheinenden Akt); l'intention est là, mais non pas avec elle l'objet lui-même, qui est, en fin de compte, destiné à la remplir ${ }^{34}$.

Le propre de la perception adéquate est en effet de supprimer toute distorsion entre l'objet phénoménal et le contenu remplissant : l'objet apparaît dans le contenu remplissant. L'intuitionnisme de Husserl trouve ainsi dans la description de la perception à la fois un terrain d'élection et un modèle généralisable: tout acte remplissant peut être nommé en un sens élargi «perception ». Les actes de signification constituent alors la sphère des actes de pensée impropres tandis que les actes d'intuition constituent la sphère des actes de pensée propres. Un acte de pensée propre est ainsi en fait un acte de perception « en un sens élargi », valant comme remplissement d'un acte de pensée impropre :

[...) les actes de pensée au sens impropre seraient les intentions de signification des énoncés et [...] tous les actes de signification qui peuvent éventuellement servir de parties de ces intentions prédicatives lles actes de pensée propresl seraient les remplissements correspondants ; donc, les intuitions d'états de choses ISachverhaltsanschauungenl et toutes les intuitions qui peuvent remplir la fonction de parties éventuelles d'intuitions d'états de choses ${ }^{35}$.

Cette homogénéité de la fonction de remplissement permet de qualifier de perception tout acte remplissant

33. Ibid., VI, I ${ }^{\mathrm{e}}$ éd., P. 776 , tr. fr., p. 289.

34. Ibid.

35. Ibid., §63. p. 722, tr. fr., p. 321-322. 
I...I sur le mode de la présentation confirmative lin der Weise der bestätigenden Selbstdarstellungl de la chose elle-même l...l et d'objet son corrélat intentionne ${ }^{36}$.

C'est donc, en fin de compte, toute la classe des actes objectivants qui tombe sous la distinction intention / remplissement, alors même que les intentions et remplissements en jeu ne concernent pas la sphère de l'expression [Aussagel. De même, les actes non objectivants sont aussi des intentions susceptibles d'un remplissement correspondant. D'une façon générale, les types d'actes intentionnels sont distingués en fonction de leur mode spécifique de remplissement. Ainsi, à toute intention, tant des actes objectivants que non objectivants, correspond un remplissement possible, et tout remplissement est une identification de l'objet visé dans l'intention à l'objet donné dans le remplissement de l'intention. On s'attendrait donc à ce que le remplissement ne puisse pas être lui-même une intention. Husserl affirme cependant que ces intuitions remplissantes sont des intentions dites intuitives : les intentions de remplissement [Wahmehmungsintentionenl sont des composantes des actes remplissants et les « synthèses de remplissement » consistent dans la coïncidence entre ces intentions perceptuelles présentes tant dans les actes intentionnels que dans les actes remplissants. En supposant que chaque acte de perception combine des intentions remplies et non remplies, la question se pose de savoir ce que sont ces intentions en tant que telles, c'est-à-dire indépendamment de leur remplissement. Husserl précise que les différences entre actes objectivants se ramènent aux intentions élémentaires et aux remplissements dont ils se constituent :

Du côté des intentions ne restent ensuite à titre de différences ultimes que les différences existant entre les intentions indicatives Isignitiven Intentionenl en tant qu'intentions par contiguité, et les intentions imaginatives en tant qu'intentions par analogie I...I Du côtè du remplissement, ce sont à nouveau, en partie, des intentions de l'une et l'autre espèce qui figurent comme composantes ; mais, sous certaines conditions Icomme dans le cas de la perception], celles également qui ne méritent plus d'être appelées intention ldie nicht mehr als Intentionen anzusprechen sind] : des composantes qui ne sont que remplissement, sans plus exiger pour elles-mêmes de remplissement l...I des présentations de l'objet lui-même visé par elles [Selbstdarstellungen des von ihnen gemeinten Objektes ${ }^{37}$. 
Il est donc clair que ce que Husserl nomme intentions perceptives comme composantes des remplissements, sont en fait des intentions indicatives [signitiven] et imaginatives: les composantes purement remplissantes ne peuvent plus être dites des intentions. Mais Husserl maintient pourtant que les intuitions sont des intentions - ces intentions intuitives présentant l'objet au sens plein [im prägnantem Sinnel.

L'introduction des noemata ne modifiera pas cette identification de l'objet de pensée et de l'objet de perception. Dans les Ideen, en effet, le noema est d'abord proposé comme corrélat des actes de perception ${ }^{38}$, et ensuite appliqué aux actes judicatifs ${ }^{39}$. Le sens objectif [gegenstandlicher Sinn] du noème perceptuel n'est autre que l'objet intentionnel comme tel " pris selon son mode "objectif" [objektivenl d'être donné ${ }^{40}$ - sens objectif « décrit avec des expressions purement objectives $»^{4 \mathrm{I}}$. Le sens noématique n'inclut rien qui n'《 apparaisse réellement » [wirklich erscheint $\gg]$ dans la chose, avec la mème façon de se présenter [Gegebenheitsweise ${ }^{42}$, de sorte qu'il est isomorphe à celle-ci, en tant que chose perçue. Dans ces conditions, les noemata de perception ne peuvent pas être des contenus conceptuels. Par exemple, à la couleur comme contenu hylétique Husserl oppose la couleur noématique ou « objective » qui s'esquisse dans le moment hylétique du vécu. Le sens du noème perceptuel de couleur est bien abstrait, c'est-à-dire qu'il est dependant de l'acte, mais il ne peut pas être conceptuel. Husserl écrit : « Cette couleur, mise entre parenthèses, appartient désormais au noème $>^{43}$; mais, certainement, cette couleur, aussi noématiquement modifiée qu'on voudra, ne peut pas être un mode de présentation conceptuel : ce doit être un mode de présentation perceptuel de la surface colorée visée par l'acte de perception. Naturellement, la reconnaissance de l'identité d'une couleur implique la possession du concept de couleur, mais il s'agit alors d'un jugement de recognition, non plus d'un acte de perception pure.

\footnotetext{
38. Husserl, Ideen, op. cit., $§ 87^{-92}$.

39. Ibid., § $93-94$.

40. Ibid., $\S 102$, p. 213 , tr. fr., p. 353 .

4I. Ibid., \$ 99, p. 250, tr. fr., p. 346.

42. Ibid., $\S 89$, p. 222, tr. fr., P. 309 .

43. Le texte allemand dit « Diese Farbe, nun in der Klammer gesetzt, gehört zum Noema », ibid., §97. p. 243, tr. fr., 337.
} 


\section{III}

Une situation de perception visuelle doit impliquer I) l'objet de la perception visuelle (la chose physique qui est vue), iI) le sujet de la situation visuelle (celui qui voit), in) le contenu sensible qui est présenté (la sensation ou apparence) iv) l'acte psychologique du sujet (son jugement), et v) le contenu propositionnel de cet acte (ce que le sujet juge ${ }^{44}$. Les auteurs qui introduisent la notion de noema ou celle de représentation mentale pour résoudre le problème de l'intentionnalité confondent la sensation ou apparence et le contenu propositionnel, c'est-à-dire ce que Wittgenstein distingue comme une condition [Zustand] et un acte [Handlungl du sujet ${ }^{45}$. Fodor écrit ainsi :

On peut présumer que la perception et la pensée sont intentionnelles dans le même sens, et il est donc probable qu'une sémantique qui ne vaut que pour la première vaut pour une mauvaise raison. Dans la perception il y a généralement coïncidence entre ce à propos de quoi un état cognitif transmet une information et ce qu'il représente [... Mais l'intentionnalité de la pensée montre que cette coïncidence n'est pas essentielle au contenu ${ }^{46}$.

Les théories naturalistes des contenus individuent les états intentionnels fondamentalement par référence à la perception, c'est-à-dire dans un champ d'entités concrètes qui sont causes des états mentaux d'un sujet. De tels états sont alors considérés comme représentationnels des entités existantes qui les causent, et la question qui divise les théories naturalistes est de savoir si le caractère représentationnel de ces états leur est intrinsèque ou extrinsèque. Dans les deux cas, c'est dans la relation du sujet au monde naturel que le problème peut recevoir une solution. Searle, qui soutient la première conception, admet que

44. R. Chisholm, « Art, Content and the Duck-Rabbit », dans R. Haller \& J. Brandl (dir.), Wittgenstein, eine Neubewertung, Vienne, Verlag Hölder, I99o, p. 64.

45. Wittgenstein, Remarks on the Philosophy of Psychology, Oxford, Blackwell, ig8o, vol. I. p. 2.

46. J. A. Fodor, A theory of Content, Cambridge, M.I.T. Press, 1990, p. 82.

46. J. Searle, Intentionality, Cambridge Mass., Cambridge University Press, 1983, p. I59, tr. fr., Paris, Minuit, 1985. Voir aussi Barry Stroud, « The Background of Thought », dans Le Pore \& Van Gulick (dir.), J. Searle and his Critics, Oxford, Blackwell, 1990, p. 245 $s q$. 
|...] le fait même d'avoir des représentations peut exister seulement sur un Arrière-plan qui confère aux représentations le caractère de "représenter quelque chose ${ }^{4} 7$.

Dretske, qui soutient la seconde conception estime que ${ }^{48}$

I...। nous aurons fait quelques progrès vers la démonstration de la pertinence causale de l'esprit l...l si nous conférons une plausibilité à l'idée selon laquelle, bien que ce peuvent être des propriétés intrinsèques au cerveau qui opèrent dans le déclenchement du comportement corporel, ce sont les propriétés extrinsèques de ce dernier qui sont pertinentes dans la structuration de ce même comportement. Ceci montrera à tout le moins qu'il n'y a rien concernant le caractère extrinsèque du mental qui le disqualifie à jouer un rôle causal dans l'explication du comportement.

Dans sa préface éditoriale à l'ouvrage cité au début de cet article, Dreyfus a très bien éclairé ces enjeux ${ }^{49}$. Il distingue trois moments essentiels dans l'histoire moderne de l'intentionnalité : celui du Husserl des Recherches logiques, celui du Husserl des Ideen, et celui de la phénoménologie existentielle du premier Heidegger et de Merleau-Ponty ${ }^{50}$. Dans la perspective du naturalisme, le premier moment correspond au réalisme, et le second à

47. J. Searle, Intentionality, Cambridge, Cambridge University Press, Ig83. p. I59-

48. F. Dretske, « Mental Events as Structuring Causes » dans Heil \& Mele (dir.), Mental Causation, Oxford, Oxford University Press, 1993. p. 131. Dretske affirme cependant soutenir la thèse d'une intentionnalitè intrinsèque : « IJ lintentionmalité que nous cherchons est une intentionnalité intrinsèque (ou, comme certains préférent dire, originale) parce que, bien que la signification soit dérivée des relations extrinseques d'une structure à d'autres choses, aucune de ces relations extrinséques n'est IJ elle-même sémantique 》. F. Dretske, «Replies », dans McLaughlin, Dretske and his Critics, Cambridge Mass., Blackwell, ig9i, p. 205.

49. H. Dreyfus, op.cit. p. 1-27.

50. Heidegger reprochait comme on sait à Husserl ce que celui-ci reprochait lui-même à Brentano, c'est-à-dire la mécompréhension de la structure de directionalité et d'intention de la conscience ldie Struktur des Sichrichtens-auf, die intentiol, Heidegger. Grundprobleme der Phänomenologie, Gesamtausgabe Band 24. Frankfort, Vittorio Klostermann, 1979, p. 89. Mais si, comme on a vu, Husserl inscrit cette directionalité dans la structure de la perception. Heidegger la rapporte à la structure de l'agir comme projet et transcendance. Le même texte poursuit : « Die Mißdeutung liegt in einer verkehrten Subjektivisierung der Intentionalität. Man setzt ein Ich, ein Subjekt an und läßt dessen sogenannter Sphäre, in die seine intentionalen Erlebnisse gleichsam eingekapselt sind ». Heidegger donne à cet égard une interprétation volontariste de l'intentio médiévale : « Die Scholastik spricht von der intention des Willens, der voluntas, d.h. sie spricht von ihr nur bezüglich des Willens » (Ibid., p. 8I). Cet usage augustinien n'est pourtant pas le plus général, même chez Thomas d'Aquin. Sur la théorie thomiste de l'intentio comme attribut ou universel par lequel la chose est connue, voir l'article de Ausonio Marras, « Scholastic Roots of Brentano's Conception of Intentionality » dans L. McAlister (dir.), The Philosophy of Brentano, Oxford, Duckworth, 1976. p. 128139 . 
l'idéalisme. Dreyfus les compare très justement respectivement aux positions de Searle et de Fodor ${ }^{51}$. Bien que Dreyfus ne nomme pas expressément le troisième moment de cette manière, c'est bien au néo-pragmatisme au sens de Rorty qu'il convient de le rapporter, moment qui associe Heidegger, Dewey et Wittgenstein dans leur commune critique de la notion d'esprit comme intériorité. Comme le dit Wittgenstein, il n'y a d'intérieur qu'à l'extérieur :

Ce qui se passe en soi-même n'a de signification que dans le cours de la vie [...] si le jeu d'expression |Ausdrucksspiell se développe, je puis sans doute dire qu'un esprit ISeelel, un intérieur se développe. Mais alors l'intérieur n'est plus la cause de l'expression ${ }^{52}$.

Dreyfus a peut-être raison lorsqu'il soutient qu'une naturalisation conséquente de l'intentionnalité devrait orienter vers la perspective du premier Heidegger, pour lequel l'intentionnalité n'est plus primitive mais dérivée d'une structure ontologique plus fondamentale. A contrario, on peut aussi estimer qu'un retour à l'essentialisme de l'école de Brentano serait justifié pour préserver une vue correcte de l'intentionnalité des pensées :

I...I si nous assumons qu'une personne peut intentionnellement se rapporter elle-même à des propriètés len bref, si nous assumons qu'une personne est rationnelle, nous n'avons plus alors besoin de la pourvoir d'un artifice de référence lbuilt-in-reference-makerl comme un noema ou un « système interne de représentation ${ }^{53}$.

\section{I.U.F.M. de Franche-Comté}

51. À l'époque où ce dernier défendait le solipsisme méthodologique.

52. Wittgenstein, Last Writings on the Philosophy of Psychology, Oxford, Blackwell, 1982, §94.

53. R. Chisholm, « Brentano and Marty on Content », op. cit., p. 8. 